\section{Lack of standardisation of ANA and implications for drug development and precision medicine}

The recent article by Pisetsky et $a l^{1}$, showing data derived from a comparison between different antinuclear antibody (ANA) assays in a cohort of patients with established systemic lupus erythematosus (SLE), addresses several important and current aspects of ANA detection. In addition, the study touches on clinical trials for ANA-associated rheumatic diseases (AARDs) and raises several relevant points that will be discussed in this letter.

\section{WHAT IS AN ANA?}

One of the fundamental questions around ANA testing is: "What is an ANA and what are diagnostic tests actually measuring?". In other words, there is no clear definition of what is and what should be included in ANA testing. ${ }^{2}$ For example, technically antibodies to cytoplasmic antigens do not belong to ANA, but can help in the diagnosis of certain AARDs and are in some countries reported as ANAs. This is important in the context of SLE as about $15 \%-30 \%$ of patients with SLE have anti-ribosomal antibodies that typically present with a cytoplasmic staining pattern. However, the cytoplasmic pattern need to be clearly defined as patients with other autoimmune diseases might also present with a cytoplasmic pattern (eg, myositis or autoimmune liver disease), although with different staining pattern. ${ }^{3}$

\section{METHODS FOR ANA DETECTION AND SENSITIVITY AND SPECIFICITY OF ANA TESTING}

Although the ANA indirect immunofluorescence (IIF) test has been recommended as the method of choice, ${ }^{4}$ the method is not without limitations. ${ }^{5}$ In addition, novel solid phase assays (SPAs) have significantly improved ${ }^{6}$ and are increasingly being used as the screening test of choice in highthroughput laboratories for the detection of ANA. ${ }^{5}$ However, it remains a matter of debate whether or not SPA such as the multiplex used in the present study should be regarded as an ANA screen or more as a screening assay for AARD (with a clear distinction in the name). The performance of IIF versus SPA also depends on the autoimmune disease under consideration in the clinic. In two recent studies on a large population of patients, it was demonstrated that IIF on HEp-2 cells performs better for systemic sclerosis, but SPAs are superior for Sjogren's syndrome $(\mathrm{SjS})$ and inflammatory myositis $^{78}$ Lastly, Bossuyt and Fieuws ${ }^{9}$ showed that adding a SPA to the IIF HEp-2 testing algorithm increased the diagnostic utility for SLE, SjS (all samples on both assays) and SSc (all samples by IIF and positives by SPA). Since IIF on HEp-2 cells lacks sensitivity for several clinically relevant autoantibodies including but not limited to SS-A/Ro60, Ro52/ TRIM21, ribosomal P and Jo- $1^{2}$ and SPAs contain a limited number of antigens, it is not surprising that the agreement is limited. Furthermore, between $10 \%$ and $20 \%$ of apparently healthy individuals have been reported to be ANA IIF positive with an established association with antibodies to DFS70, ${ }^{25}$ as well as other unknown targets. It is not unlikely that this $10 \%-20 \%$ of 'false positive' rate also occurs in patients with SLE, but will be considered true positive as it fits well to the disease state. However, those patients might have a different clinical phenotype and also require different clinical care. One potential strategy is to define ANA positivity for clinical trial enrolment (and drug prescription) by a positive result in different methods (eg, SPA and IIF).

\section{STANDARDISATION EFFORTS}

Despite efforts and advances in the field of ANA test standardisation (mostly driven by the International Consensus on ANA Pattern ${ }^{3}$ ), ANA testing in clinical practice remains challenging. One major area of discussion is the screening dilution used for ANA by IIF, which is directly linked to the sensitivity of the assay. Recently, the new SLE criteria were published recommending a screening dilution of $1: 80$ versus the $1: 40$ used in the present study. ${ }^{10}$ Using 1:80, potentially even more samples might have been negative in the study by Pisetsky et $a{ }^{1} .^{1}$ Although the serum dilution is relevant, other factors such as the conjugate strength and specificity, stringency of washing steps as well as the microscope light sources and optics play an important role in the variability. The sensitivity of slides from different manufacturers differ not only in terms of the overall sensitivity butalso in regards to the analytical sensitivity of the individual ANA fine-specificities. ${ }^{11}$ These differences are attributed to the way the cells are grown, immobilised and fixed on the slides. Ideally, monospecific patient samples and/or human monoclonal antibodies should be used in titration studies to fully assess the analytical sensitivity by fine-specificity for all manufacturers. Preliminary data are available for anti-Rib-P antibodies, which show significant inter-manufacturer sensitivity variations. ${ }^{11}$ Besides the slides and other reagents, a very important aspect for the detection of ANA is the subjectivity in interpretation. ${ }^{5}$ Although the study by Pisetsky et al used trained technicians in a single laboratory, interobserver variability was not clearly addressed, a very important factor as reported in a recent study. ${ }^{12}$ Consequently, automated interpretation systems (available from several manufacturers) are highly recommended to reduce variability and subjectivity, which is of particular importance in a global clinical trial setting. This also facilitates unbiased image acquisition and documentation of results, which is also important for clinical trials.

\section{REPORTED DISCORDANCE OF METHODS}

The number of patients included in this study ${ }^{1}$ was small $(n=103)$, and no confidence intervals (CIs) for the frequency of negative results were provided, which makes it difficult to fully assess the level of disagreement of the individual assays. In more detail, ANA negativity in the 103 patients with SLE ranged widely from $4.9 \%$ to $22.3 \%$, but was also accompanied by large 95\% CIs (IIF Kit 1=22.3\% (14.9\%-31.1\%), IIF Kit 2=9.7\% (4.2\%-15.8\%), IIF Kit 3 4.9\% (0.8\%-9.2\%), ELISA $11.7 \%$ (5.7\%-18.3\%), Multiplex 13.6\% (7.3\%-20.7\%)). Therefore, taking into account CIs, only the difference between IIF Kits 1 and 3 showed significance. However, the key message that there is variation among ANA assays is not altered as other studies have shown similar results. ${ }^{11}$

\section{LUPUS AS A HETEROGENEOUS GROUP OF SYMPTOMS}

It is widely appreciated that SLE can manifest in various forms and that autoantibody profiles can subdivide patients into more homogeneous groups. ${ }^{13-15}$ Also, it is possible that stratification of patients powered by machine learning techniques will lead to a novel, molecular-based nomenclature of disease that will likely improve patient outcome. ${ }^{16}$ For clinical trials in patients with AARD, ANA, even if clearly defined, is unlikely to provide the full insight into meaningful disease subsets of patients who respond to a particular treatment. Along those lines, autoantibodies might not provide a robust reflection of pathogenic pathways where other biomarkers such as cytokines, inflammatory 
proteins or complement components can provide further insights into potential treatment strategies. ${ }^{15}$

\section{COMPANION OR COMPLEMENTARY DIAGNOSTICS}

As pointed out by Pisetsky et al, biomarkers have significant potential to help stratify patients with SLE into more meaningful subsets and are often referred to theranostic biomarkers. From a practical and regulatory perspective, it is important to clearly differentiate between companion and complementary diagnostics. Companion diagnostics have to be included into early clinical trials and will eventually become a prerequisite for the associated drug and are therefore listed in the drug label. Since the test has to be part of the regulatory submission, only the tests that have been included might be used. By contrast, complementary diagnostics can be established after the commercialisation of a drug and 'only' rely on the in vitro diagnostic regulation.

\section{CONCLUDING REMARKS}

Taken together, the report by Pisetsky et al touches important aspects in the context of ANA testing and the high visibility of this article will hopefully trigger new initiatives for better understanding of the variability of ANA tests and the consequences. Ideally, those initiates should include rheumatologists, autoimmunologists, standardisation organisations as well as experts from diagnostic and pharmaceutical companies. Such an approach could lead to more precise and commutable testing, improved clinical trials, reduced healthcare expenditures and ultimately to better patient care and outcome.

\section{Michael Mahler}

Department of Research, Inova Diagnostics, San Diego, California, USA

Correspondence to Dr Michael Mahler, Department of Research, Inova Diagnostics, San Diego, CA 92131, USA; mmahler@inovadx.com

Competing interests $\mathrm{MM}$ is an employee of Inova Diagnostics.

Patient consent Not required.

Provenance and peer review Not commissioned; internally peer reviewed.

(c) Article author(s) (or their employer(s) unless otherwise stated in the text of the article) 2019. All rights reserved. No commercial use is permitted unless otherwise expressly granted.

$$
\text { A) Check for updates }
$$

To cite Mahler M. Ann Rheum Dis 2019;78:e33.
Received 7 March 2018

Accepted 11 March 2018

Published Online First 24 March 2018

\section{Linked}

http://dx.doi.org/10.1136/annrheumdis-2018-213399

Ann Rheum Dis 2019:78:e33. doi:10.1136/annrheumdis-2018-213374

REFERENCES

1 Pisetsky DS, Spencer DM, Lipsky PE, et al. Assay variation in the detection of antinuclear antibodies in the sera of patients with established SLE. Ann Rheum Dis 2018;77:911-3.

2 Agmon-Levin N, Damoiseaux J, Kallenberg C, et al. International recommendations for the assessment of autoantibodies to cellular antigens referred to as anti-nuclear antibodies. Ann Rheum Dis 2014;73:17-23.

3 Chan EK, Damoiseaux J, de Melo Cruvinel W, et al. Report on the second International Consensus on ANA Pattern (ICAP) workshop in Dresden 2015. Lupus 2016;25:797-804.

4 Meroni PL, Schur PH. ANA screening: an old test with new recommendations. Ann Rheum Dis 2010;69:1420-2.

5 Mahler M, Meroni PL, Bossuyt X, et al. Current concepts and future directions for the assessment of autoantibodies to cellular antigens referred to as anti-nuclear antibodies. J Immunol Res 2014:2014:1-18.

6 Bentow C, Lakos G, Rosenblum R, et al. Clinical performance evaluation of a novel, automated chemiluminescent immunoassay, QUANTA Flash CTD Screen Plus. Immunol Res 2015;61:110-6.

7 van der Pol P, Bakker-Jonges LE, Kuijpers J, et al. Analytical and clinical comparison of two fully automated immunoassay systems for the detection of autoantibodies to extractable nuclear antigens. Clin Chim Acta 2018;476:154-9.

8 Claessens J, Belmondo T, De Langhe E, et al. Solid phase assays versus automated indirect immunofluorescence for detection of antinuclear antibodies. Autoimmun Rev 2018.

9 Bossuyt X, Fieuws S. Detection of antinuclear antibodies: added value of solid phase assay? Ann Rheum Dis 2014:73.

10 Tan EM, Feltkamp TE, Smolen JS, et al. Range of antinuclear antibodies in "healthy" individuals. Arthritis Rheum 1997:40:1601-11.

11 Mahler M, Ngo JT, Schulte-Pelkum J, et al. Limited reliability of the indirect immunofluorescence technique for the detection of anti-Rib-P antibodies. Arthritis Res Ther 2008; 10:R131.

12 Rigon $\mathrm{A}$, Infantino $\mathrm{M}$, Merone $\mathrm{M}$, et al. The inter-observer reading variability in antinuclear antibodies indirect (ANA) immunofluorescence test: a multicenter evaluation and a review of the literature. Autoimmun Rev 2017;16:1224-9.

13 Artim-Esen B, Cene E, Sahinkaya Y, et al. Cluster analysis of autoantibodies in 852 patients with systemic lupus erythematosus from a single center.J Rheumatol 2014:41:1304-10.

14 To $\mathrm{CH}$, Petri M, Ch T. Is antibody clustering predictive of clinical subsets and damage in systemic lupus erythematosus? Arthritis Rheum 2005:52:4003-10

15 Pacheco Y, Barahona-Correa J, Monsalve DM, et al. Cytokine and autoantibody clusters interaction in systemic lupus erythematosus. J Trans/ Med 2017:15:239.

16 Barturen G, Beretta L, Cervera R, et al. Moving towards a molecular taxonomy of autoimmune rheumatic diseases. Nat Rev Rheumatol 2018;14:75-93. 